\title{
Inhibitory effect of taspine derivative TAD1822-7 on tumor cell growth and angiogenesis via suppression of EphrinB2 and related signaling pathways
}

\author{
RUI LIU \\ RUNZE YU \\ YUXIN CUI \\ MENGYING FAN \\ BO WANG \\ YANMIN ZHANG* \\ School of Pharmacy, Xi'an Jiaotong \\ University, $710061 \mathrm{Xi}^{\prime}$ an, Shaanxi \\ China
}

Accepted December 3, 2018

Published online December 22, 2018

\begin{abstract}
The aim of this study was to investigate the inhibitory effect of TAD1822-7, a synthesized taspine derivative, on cancer through its effects on tumor cell growth and angiogenesis via suppression of EphrinB2. The obtained data showed that TAD1822-7 decreased Bel-7402 cell viability and colony formation ability and suppressed cell migration. TAD1822-7 effectively inhibited blood vessel formation in an aortic ring assay to examine angiogenesis. Moreover, it also downregulated the expression of VEGFR2, VEGFR3, CD34, PLC $\gamma$, Akt, MMP2, MMP9, and CXCR4, and suppressed the expression of EphrinB2 and its PDZ protein, PICK1, in Bel-7402 cells. These results indicate that TAD1822-7 is a potential antiangiogenic agent that can inhibit the viability and migration of Bel-7402 cells via suppression of EphrinB2 and the related signaling pathways.
\end{abstract}

Keywords: TAD1822-7, EphrinB2, Bel-7402 cells, proliferation, migration, anti-angiogenesis

Hepatocellular carcinoma (HCC) is one of the common malignant cancers with high morbidity and mortality (1). Ephrins are the ligands for erythropoietin-producing hepatoma amplified sequence (Eph) family receptor tyrosine kinases (RTKs), which are membrane-bound proteins (2). EphrinB2 is a subtype of EphrinB, which is a ligand for the EphB2, EphB3, and EphB4 kinases, and the only known ligand of EphB4 is EphrinB2 (3). Ephrin-mediated interactions between EphrinB2 and EphB4 are essential for tumor angiogenesis (4).

Angiogenesis plays a pivotal role in tumor progression, including primary capillary germination, branching and remodeling, or forming a mature vascular network (5). Stimulation of EphrinB2, which phosphorylates its cognate receptor EphB4, provides signals for cell survival and migration in cancers (6). Meanwhile, the upregulation of EphrinB2 can activate VEGF/VEGFR signaling during tumor formation, which leads to activation of the PI3K/Akt and PLC- $\gamma /$ PKC pathways and other related downstream protein expressions (7).

\footnotetext{
* Correspondence, e-mail: zhang2008@mail.xjtu.edu.cn
} 
Taspine has been reported to have antitumor activity (8), and TAD1822-7 (Fig. 1a) is one of its derivatives, designed and synthesized as a lead compound containing a biphenyl scaffold for better tumor suppression effects (8). However, no activity of TAD1822-7 against HCC has ever been reported and the related mechanism is still unknown. The current study is focused on the inhibitory effects of TAD1822-7 on HCC and the involved mechanisms.

\section{EXPERIMENTAL}

\section{Materials}

Taspine derivative TAD1822-7 was synthesized at the School of Pharmacy, Xi'an Jiaotong University (8). EphB4 Fluorescence-chimera (B4-Fc) was purchased from Beijing Biotechnology Co., Ltd. (MDL No. MFCD01867528, China). DAPI, MTT, RPMI-1640 medium and Dulbecco's Modified Eagle's medium (DMEM) were purchased from Sigma-Aldrich (USA). Thrombin was obtained from Guoao Pharmaceutical (China). Fetal bovine serum (FBS) was purchased from HyClone (USA). G418 was obtained from Gibco (USA). RIPA lysis buffer was purchased from Beijing BLKWB Biotechnology Co., Ltd (China). Enhanced Chemiluminescence (ECL) Plus Reagent kit was obtained from Pierce Biotechnology, Inc. (USA). EphrinB2 (Cat. \#6957-1), MMP9 (Cat. \#2551-1), and MMP2 (Cat. \#1948-1) antibodies were purchased from Epitomics (USA). VEGFR2 (Cat. \#26415-1-AP), VEGFR3 (Cat. \#20712-1-AP), PICK1 (Cat. \#10983-2-AP), Syntenin-1 (Cat. \#22399-1-AP), CD34 (Cat. \#14486-1-AP), CXCR4 (Cat. \#11073-2-AP), and GAPDH (Cat. \#60042-1-IG) antibodies were purchased from Proteintech Group (USA). Akt (Cat. \#4685), PLC $\gamma 1$ (Cat. \#5690), anti-mouse IgG (H+L; Cat. \#14709) and anti-rabbit IgG (Cat. \#14708) secondary antibodies were purchased from Cell Signaling Technology, Inc. (USA).

\section{Cells and animals}

Bel-7402, HepG2, A549, PANC-1, and SGC-7901 cell lines were purchased from Shanghai Institute of Cell Biology of the Chinese Academy of Science (China) and cultured in RPMI-1640 medium supplemented with 10 \% FBS. EphrinB2/HEK293 cells were constructed at Food and Drug Research \& Test Center, Xi'an Jiaotong University, and cultured in DMEM supplemented with $300 \mathrm{mg} \mathrm{mL}^{-1} \mathrm{G} 418$, penicillin/streptomycin and $10 \% \mathrm{FBS}$.

Sprague-Dawley rats (40-60 g, 2-4 weeks of age, 3 male and 3 female) were purchased from the Animal Research Center of Xi'an Jiaotong University, and were sacrificed by $\mathrm{CO}_{2}$ inhalation. The animal experimental protocol was approved by the Animal Ethics Committee of Xi'an Jiaotong University (license number: XJTULAC 2018-0542).

\section{Cell viability test}

The cell viability test was assessed by the MTT assay and cell colony survival assay. In the MTT assay, cells were treated for $48 \mathrm{~h}$ with 4, 8 and $16 \mu \mathrm{M}$ TAD1822-7 dissolved in RPMI-1640 medium and the medium was used as a control, and then incubated with MTT solution; the formed crystals were dissolved with DMSO, and the absorbance at $490 \mathrm{~nm}$ was measured using the Molecular Devices FlexStation ${ }^{\circledR} 3$ microplate reader (San Jose, CA, USA). In the colony survival assay, cells were plated at 200 cells per well in a 12-well plate 
R. Liu et al.: Inhibitory effect of taspine derivative TAD1822-7 on tumor cell growth and angiogenesis via suppression of EphrinB2 and related signaling pathways, Acta Pharm. 69 (2019) 423-431.

and treated with TAD1822-7 when the colonies were visible and countable. Colonies were fixed in methanol, stained with crystal violet, and observed under a microscope.

\section{EphB4 fluorescence-chimera (B4-Fc) binding to EphrinB2}

Binding ability of B4-Fc to EphrinB2 on the cytomembrane of Bel-7402 was assessed by EphrinB2 expression analysis. Bel-7402 cells were seeded in 96-well plates, incubated with $1 \mathrm{mg} \mathrm{mL}^{-1} \mathrm{~B} 4-\mathrm{Fc}$ dissolved in RPMI-1640 medium for $4 \mathrm{~h}$ at $37^{\circ} \mathrm{C}$ and observed using a FITC fluorescence microscope. To determine the target role of TAD1822-7 on EphrinB2, HEK293/EphrinB2 cells were seeded in 96-well plates and co-cultured with $1 \mathrm{mg} \mathrm{mL}^{-1} \mathrm{~B} 4-\mathrm{Fc}$ and 4, 8 and $16 \mu \mathrm{M}$ TAD1822-7 dissolved in DMEM for $6 \mathrm{~h}$ at $37^{\circ} \mathrm{C}$; the medium was used as a control. After that, the cells were washed twice with PBS, fixed in $4 \%$ paraformaldehyde (PFA) for $15 \mathrm{~min}$, stained with $1 \mu \mathrm{g} \mathrm{mL} \mathrm{m}^{-1} \mathrm{DAPI}$ for $15 \mathrm{~min}$, and observed using a fluorescence microscope.

\section{Cell migration test}

The cell migration test was performed using the Transwell system, in which there is an 8-mm pore of Millicell polycarbonate membrane for the cells to migrate through. Serum-starved Bel-7402 cells were plated at a density of $1 \times 10^{4}$ cells per well in the upper chamber of the system and treated with TAD1822-7. After migration, the cells on the lower surface were fixed, stained with $0.2 \%$ crystal violet and observed under a microscope.

\section{Aortic ring culture}

Dissected thoracic aortas from Sprague-Dawley rats were transferred immediately to PBS, the adventitia was removed, and the small vessels around the aorta were carefully transversely truncated with $1 \mathrm{~mm}$ cuts and cultured in polymerized fibrinogen with thrombin dissolved in DMEM in 48-well plates. More polymerized fibrinogen with thrombin was put on the aortic ring, and the aortic ring was cultured in the sandwich of the medium. After 7 days, the sprouting vessels were observed under a stereomicroscope and treated for $48 \mathrm{~h}$ with 4,8 and $16 \mu \mathrm{M}$ TAD1822-7 in DMEM and DMEM was used as a control. Neo-microvessels were observed and imaged throughout the experiment.

\section{Western blotting analysis}

After treatment with 0, 4, 8 and $16 \mu \mathrm{M}$ TAD1822-7 for $48 \mathrm{~h}$, the proteins in Bel-7402 cells were extracted using RIPA lysis buffer, denatured, separated by electrophoresis, and transferred to polyvinylidene fluoride membranes. After incubation with the corresponding primary antibodies and secondary antibodies, the membranes containing the proteins were exposed using an ECL kit. The Image-Pro Plus 5.1 (Rockville, MD, USA) was used for the quantification of protein expression.

\section{Statistical analysis}

Data were expressed as the mean \pm SD and a two-tailed unpaired Student's $t$-test was used to determine significance in statistical comparisons, with statistical significance 
accepted at $p<0.05\left({ }^{*} p<0.05,{ }^{* *} p<0.01\right.$, and $\left.{ }^{* * *} p<0.001\right)$. All statistical analyses were computed using GraphPad Prism 6.0 (USA).

\section{RESULTS AND DISCUSSION}

\section{TAD1822-7 inhibited cell viability and colony formation}

Cell viability of Bel-7402, HepG2, A549, PANC-1, and SGC-7901 cells treated with TAD1822-7 were investigated using the MTT assay (Fig. 1b), which revealed an inhibitory effect, especially in Bel-7402 cells. Meanwhile, when treated with 0, 4, 8 and $16 \mu$ M TAD18227 until the colonies were visible and countable, colony formation was also similarly inhibited (Fig. 1c). Hence, TAD1822-7 has a potential antitumor activity in HCC and it may be regulated by EphrinB2.

\section{TAD1822-7 and B4-Fc bind competitively to EphrinB2}

B4-Fc staining of Bel-7402 cells revealed high expression of EphrinB2 (Fig. 2a). Protein expression of EphrinB2 was effectively downregulated in both Bel-7402 cells (Fig. 2b,c) and EphrinB2/HEK293 cells (Fig. 2d,e) after the treatment of 0, 4, 8 and $16 \mu$ M TAD1822-7, which indicated that TAD1822-7 could suppress EphrinB2 expression. In addition, EphrinB2/ HEK293 cells were treated with $0.5 \mathrm{mg} \mathrm{mL}^{-1} \mathrm{~B} 4-\mathrm{Fc}$ and $0,4,8$ and $16 \mu \mathrm{M}$ TAD1822-7 simultaneously for $4 \mathrm{~h}$, and then stained with DAPI. The results showed that TAD1822-7 inhibited the binding of B4-Fc to EphrinB2 in EphrinB2/HEK293 cells (Fig. 2f), indicating that TAD1822-7 and B4-Fc could bind competitively to EphrinB2 and TAD1822-7 to inhibit EphrinB2 activation.

a)<smiles>COc1cc(-c2cc(NC(=O)Nc3ccc(OCCN4CCOCC4)cc3C)cc(OC)c2OC)ccc1/C(C)=N/O</smiles>

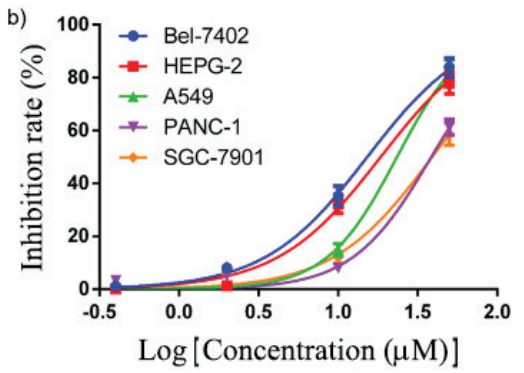

c)
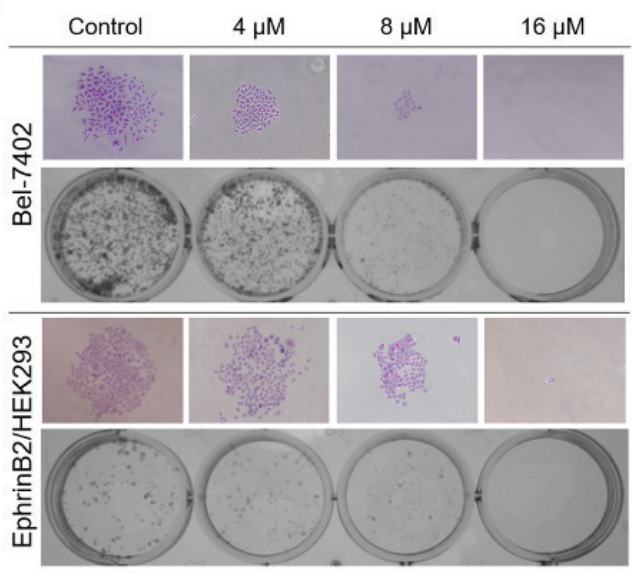

Fig. 1. TAD1822-7 suppressed the proliferation and colony formation of Bel-7402 cells and EphrinB2/ HEK293 cells. a) chemical structure of TAD1822-7, b) inhibition of TAD1822-7 on Bel-7402, HepG2, A549, PANC-1, and SGC-7901 cells, c) representative images showed colony formation of Bel-7402 cells and EphrinB2/HEK293 cells by TAD1822-7. 
R. Liu et al.: Inhibitory effect of taspine derivative TAD1822-7 on tumor cell growth and angiogenesis via suppression of EphrinB2 and related signaling pathways, Acta Pharm. 69 (2019) 423-431.

a)
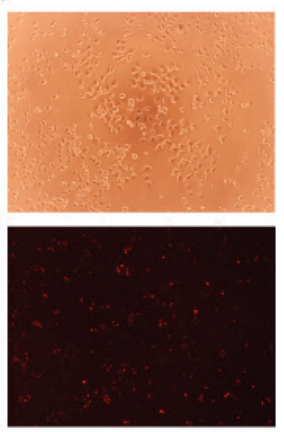

b)

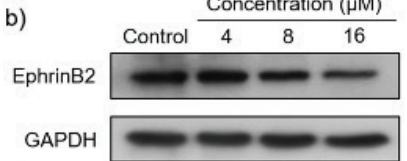

c)

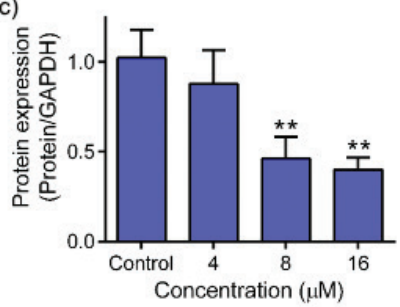

d)

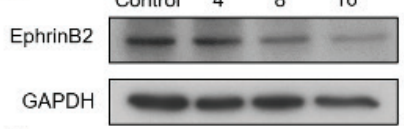

e)

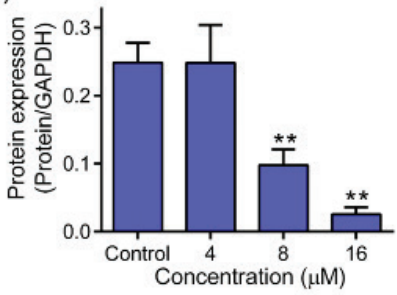

f)

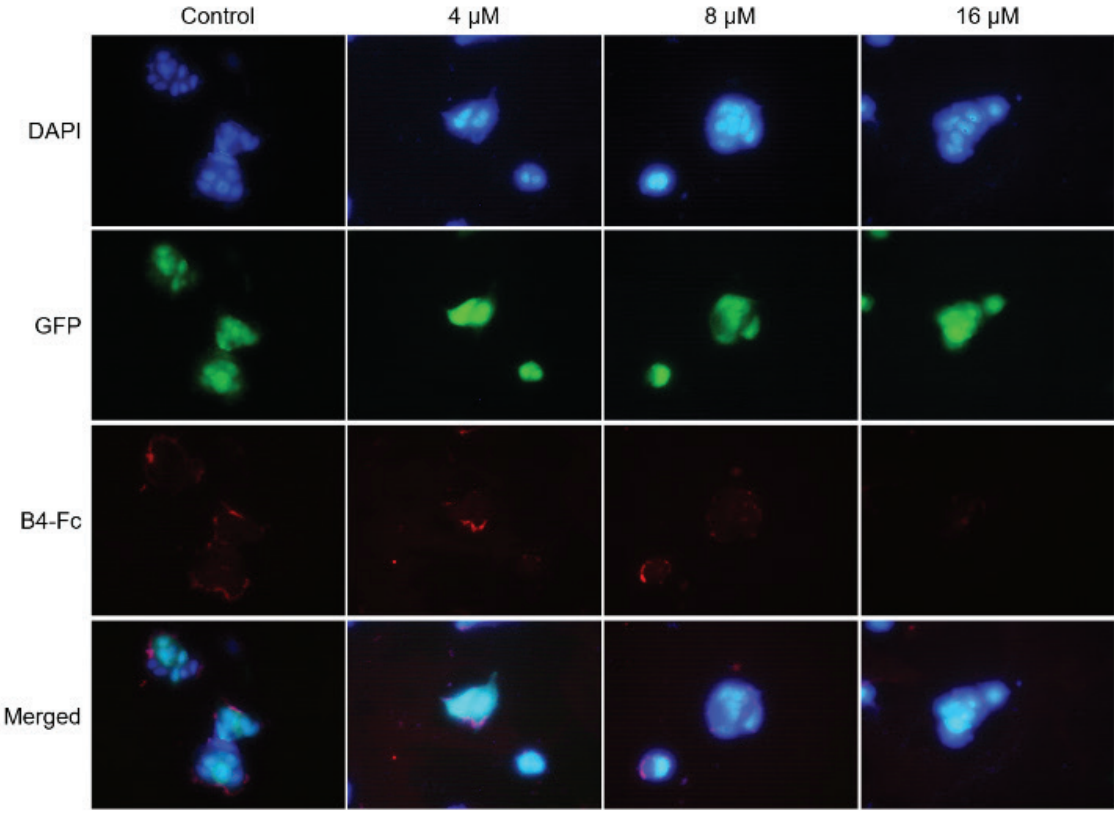

Fig. 2. TAD1822-7 inhibited the expression of EphrinB2: a) representative images of Bel-7402 cells with B4-Fc, b) Western blot analysis of EphrinB2 expression level in Bel-7402, c) quantification of IOD value for b), d) Western blot analysis of EphrinB2 expression level in EphrinB2/HEK293 cells, e) quantification of IOD value for d), f) representative images of EphrinB2/HEK293 cells treated with B4-Fc and TAD1822-7. Significant difference vs. control group: $p<0.05\left({ }^{*} p<0.05,{ }^{* *} p<0.01,{ }^{* * *} p<0.001\right)$.

\section{TAD1822-7 inhibited Bel-7402 cell migration}

TAD1822-7 dose-dependently inhibited the migration ability of Bel-7402 cells in the transwell migration assay (Fig. 3a,b). MMP2 and MMP9 are critical components in cancer cell invasion and migration (9), and CXCR4 is associated with cancer cell migration (10). In 
a)

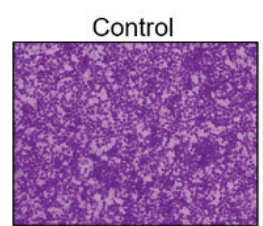

$8 \mu \mathrm{M}$

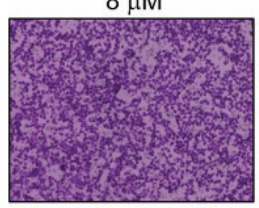

c)

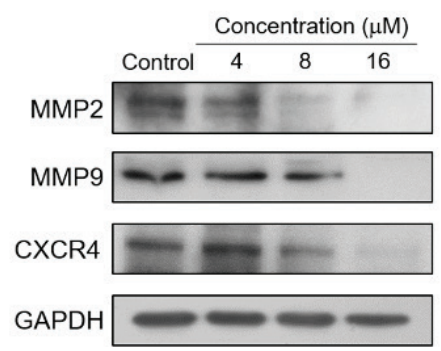

$16 \mu \mathrm{M}$ b)
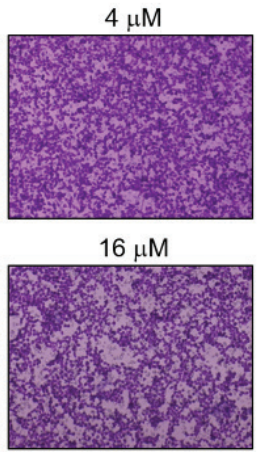

d)
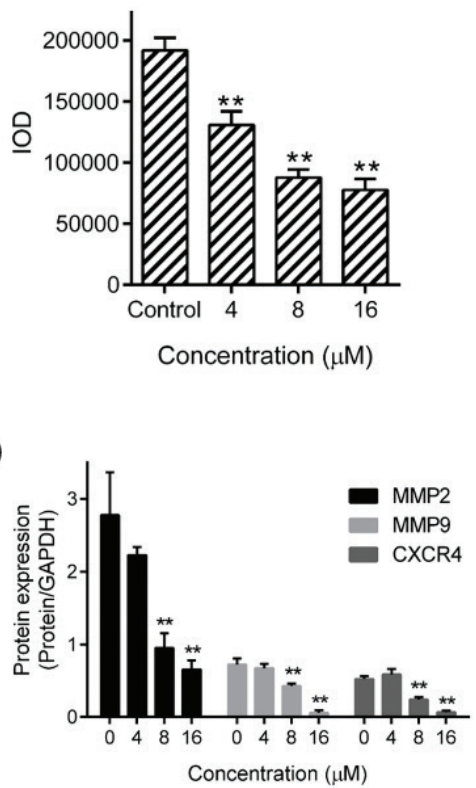

Fig. 3. TAD1822-7 suppressed Bel-7402 cell migration and the expression of MMP2, MMP9, and CXCR4: a) representative images of cells that migrated through the polycarbonate membranes in Transwell assays stained with $0.2 \%$ crystal violet (magnification, x200), b) quantification of the IOD value for cells that migrated through the polycarbonate membranes in a), c) Western blot analysis of the expression levels of MMP2, MMP9, and CXCR4 in Bel-7402 cells, d) quantification of IOD values for c), significant difference vs. control group: $p<0.05\left({ }^{*} p<0.05,{ }^{* *} p<0.01,{ }^{* * *} p<0.001\right)$.

this study, TAD1822-7 inhibited the expression of MMP2, MMP9, and CXCR4 in Bel-7402 cells in a dose-dependent manner (Fig. 3c,d), which was thought to confer the anti-migration effect.

\section{TAD1822-7 inhibited angiogenesis in the aortic ring model}

In the aortic ring assay, the entire angiogenesis process can be reproduced in vitro through the culture of rat aortic explants in a three-dimensional cell growth matrix under chemically defined growth factor conditions. After the sprouting vessels were observed, 0, 4, 8 and $16 \mu \mathrm{M}$ TAD1822-7 were added and cultured for $48 \mathrm{~h}$. The result showed that TAD1822-7 inhibited the number of sprouting vessels (Fig. 4a), which indicated an inhibitory effect on angiogenesis. Pitulescu, M.E. et al. reported that EphrinB2 was crucial in tumor angiogenesis, which can be regarded as a promising prognostic indicator and a target to modulate angiogenesis in cancer therapies (11).

CD34 protein expression is found on early hematopoietic and vascular-associated tissue and it also facilitates cell migration (12). VEGFR2 is the main mediator for the mito- 
a)
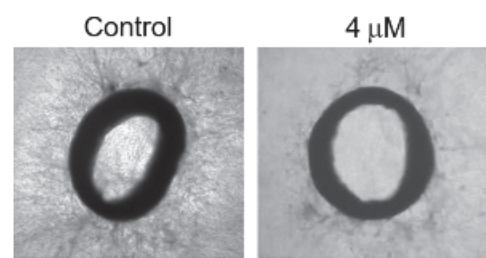

b)

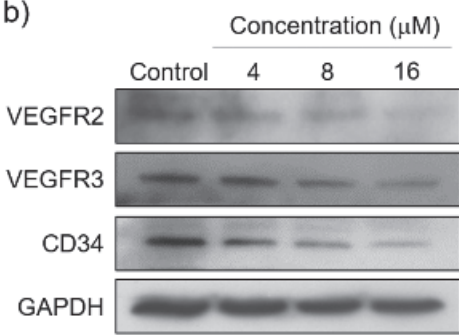

d)

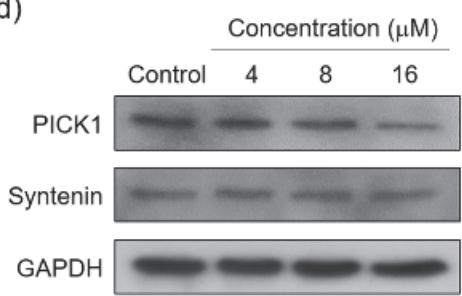

f)

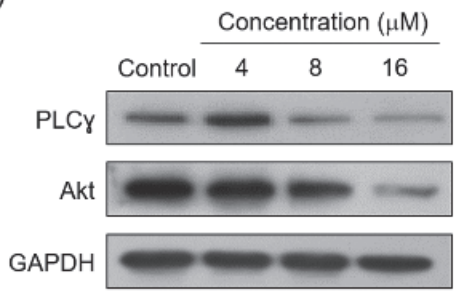

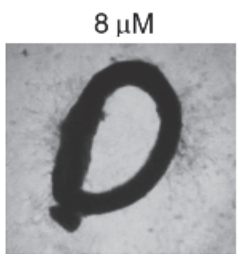

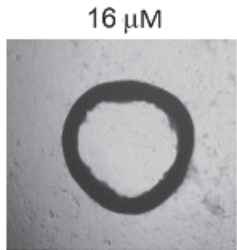

c)
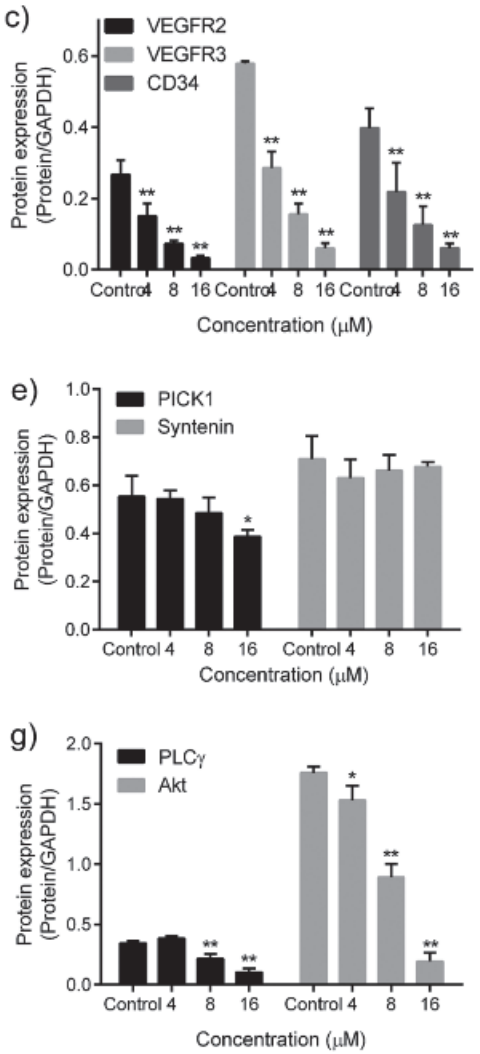

Fig. 4. The effect of TAD1822-7 on angiogenesis and the involved signal pathway proteins: a) representative images show angiogenesis of the rat aortic ring under the treatment of TAD1822-7, b) Western blot analysis of the expression levels of VEGFR2, VEGFR3, and CD34 in Bel-7402 cells, c) quantification of IOD value for b), d) effect of TAD1822-7 on PDZ-binding motif protein of EphrinB2, PICK1, and Syntenin, e) quantitation data of densitometry analysis of the bands from d), f) effect of TAD1822-7 on PLC $\gamma$ and Akt, g) quantitation data of IOD value for f), significant difference vs. control group: $p<0.05\left({ }^{*} p<0.05\right.$, $\left.{ }^{* *} p<0.01,{ }^{* * *} p<0.001\right)$.

genic, angiogenic and enhanced permeability properties of VEGF-A, and VEGFR3 is also reported to be involved in angiogenesis in cancer; the blocking of these proteins is thought to suppress angiogenic sprouting and vascular network formation (8). TAD1822-7 suppressed 
R. Liu et al.: Inhibitory effect of taspine derivative TAD1822-7 on tumor cell growth and angiogenesis via suppression of EphrinB2 and related signaling pathways, Acta Pharm. 69 (2019) 423-431.

the expression of VEGFR2 and VEGFR3 in Bel-7402 cells in a dose-dependent manner after treatment for $48 \mathrm{~h}$, and CD34 expression was also downregulated by TAD1822-7 (Fig. 4b,c).

\section{TAD1822-7 regulated EphrinB2 PDZ-binding motif protein of Bel-7402 cells}

EphrinB2 plays a crucial role in tumor angiogenesis, for which the presence of the C-terminal PDZ motif is required to allow recruitment of the related functional proteins around the EphrinB2 intracellular region. Syntenin-1 and PICK1 are the PDZ-binding motif proteins of EphrinB2, and may affect the activation of EphrinB2 (13). Expression of PICK1 was suppressed drastically by TAD1822-7, but there was a minimal effect of TAD1822-7 on Syntenin-1 (Fig. 4d,e), which indicated that TAD1822-7 inhibited the activation of EphrinB2 through its PICK1 intracellular tyrosine kinase domain.

\section{TAD1822-7 inhibited the related downstream signal pathways}

VEGFR2 is essential for many angiogenic processes in tumor vascularization that regulate the downstream signaling pathway to affect the cell function. TAD1822-7 has the ability to inhibit the protein expression of PLC- $\gamma$ and Akt (Fig. 4f,g). PLC $\gamma /$ PKC and PI3K/ Akt signaling pathways are two of the diverse molecular signaling pathways related to tumor cell growth, and the activation of these two pathways may lead to aberrant cell proliferation (14). TAD1822-7 inhibited the expression of PLC- $\gamma$ and Akt in Bel-7402 cells. Activation of VEGFR3 is known to cause PI3K-dependent activation of Akt and PKC (15). We suspected that TAD1822-7 inhibited the expression of EphrinB2, thereby suppressing the related PI3K/Akt and PLC $\gamma /$ PKC signaling pathways. Inhibition of EphrinB2 by TAD1822-7 will negatively regulate VEGFR2, VEGFR3, and CD34 to inhibit angiogenesis.

\section{CONCLUSIONS}

EphrinB2 is an angiogenesis-related transmembrane protein, which is upregulated and requires the presence of its C-terminal PDZ motif for activity in tumor cells. TAD18227 is a synthesized taspine derivative for tumor growth inhibition, which inhibits cell viability and migration in Bel-7402 cells via competitive binding to EphrinB2 and regulating EphrinB2 related signaling molecules expression. Meanwhile, TAD1822-7 was demonstrated to have the potential function of anti-angiogenesis. Furthermore, the results suggest that TAD1822-7 is a potential anti-tumor agent for hepatocellular carcinoma.

Acknowledgement. - This work was supported by the National Natural Science Foundation of China (Grant No. 81773772) and the Fundamental Research Funds for the Central Universities.

\section{REFERENCES}

1. D. S. Dizon, L. Krilov, E. Cohen, T. Gangadhar, P. A. Ganz, T. A. Hensing, S. Hunger, S. S. Krishnamurthi, A. B. Lassman, M. J. Markham, E. Mayer, M. Neuss, S. K. Pal, L. C. Richardson, R. Schilsky, G. K. Schwartz, D. R. M. A. Spriggs, Villalona-Calero, G. Villani and G. Masters, Clinical 
cancer advances 2016: Annual report on progress against cancer from the American Society of Clinical Oncology, J. Clin. Oncol. 34 (2016) 987-1011; https://doi.org/10.1200/JCO.2015.65.8427

2. N. Holder and R. Klein, Eph receptors and ephrins: effectors of morphogenesis, Development 10 (1999) 2033-2044.

3. J. E. Chrencik, A. Brooun, M. L. Kraus, M. I. Recht, A. R. Kolatkar, G. W. Han, J. M. Seifert, H. Widmer, M. Auer and P. Kuhn, Structural and biophysical characterization of the EphB4*ephrinB2 protein-protein interaction and receptor specificity, J. Biol. Chem. 38 (2006) 28185-28192; https://doi. org/10.1074/jbc.M605766200

4. S. S. Gerety, H. U. Wang, Z. F. Chen and D. J. Anderson, Symmetrical mutant phenotypes of the receptor EphB4 and its specific transmembrane ligand ephrin-B2 in cardiovascular development, Mol. Cell. 3 (1999) 403-414.

5. J. Folkman, Angiogenesis in cancer, vascular, rheumatoid and other diseases, Nat. Med. 1 (1995) 27-31; https://doi.org/10.1038/nm0195-27

6. S. R. Kumar, J. Singh, G. Xia, V. Krasnoperov, L. Hassanieh, E. J. Ley, J. Scehnet, N. G. Kumar, D. Hawes, M. F. Press, F. A. Weaver and P. S. Gill, Receptor tyrosine kinase EphB4 is a survival factor in breast cancer, Am. J. Pathol. 169 (2006) 279-293; https://doi.org/10.2353/ajpath.2006.050889

7. D. Yang, C. Jin, H. Ma, M. Huang, G. Shi, J. Wang and M. Xiang, EphrinB2/EphB4 pathway in postnatal angiogenesis: a potential therapeutic target for ischemic cardiovascular disease, Angiogenesis 19 (2016) 297-309; https://doi.org/10.1007/s10456-016-9514-9

8. C. Wang, J. Dong, Y. Zhang, F. Wang, H. Gao, P. Li, S. Wang and J. Zhang, Design, synthesis and biological evaluation of biphenyl urea derivatives as novel VEGFR-2 inhibitors, Medchemcomm 11 (2013) 1434-1438; https://doi.org/10.1039/C3MD00192J

9. Q. Y. Chen, Y. Zheng, D. M. Jiao, F. Y. Chen, H. Z. Hu, Y. Q. Wu, J. Song, J. Yan, L. J. Wu and G. Y. Lv, Curcumin inhibits lung cancer cell migration and invasion through Rac1-dependent signaling pathway, J. Nutr. Biochem. 25 (2014) 177-185; https://doi.org/10.1016/j.jnutbio.2013.10.004

10. Y. Chen, G. Stamatoyannopoulos and C. Z. Song, Down-regulation of CXCR4 by inducible small interfering RNA inhibits breast cancer cell invasion in vitro, Cancer Res. 63 (2003) 4801-4804.

11. M. E. Pitulescu and R. H. Adams, Eph/ephrin molecules - a hub for signaling and endocytosis, Genes Dev. 24 (2010) 2480-2492; https://doi.org/10.1101/gad.1973910

12. J. S. Nielsen and K. M. McNagny, Novel functions of the CD34 family, J. Cell Sci. 121 (2008) 36833692; https://doi.org/10.1242/jcs.037507

13. T. Makinen, R. H. Adams, J. Bailey, Q. Lu, A. Ziemiecki, K. Alitalo, R. Klein and G. A. Wilkinson, PDZ interaction site in ephrinB2 is required for the remodeling of lymphatic vasculature, Genes Dev. 19 (2005) 397-410; https://doi.org/10.1101/gad.330105

14. T. V. Byzova, C. K. Goldman, N. Pampori, K. A. Thomas, A. Bett, S. J. Shattil and E. F. Plow, A mechanism for modulation of cellular responses to VEGF: activation of the integrins, Mol. Cell. 6 (2000) 851-860.

15. T. Makinen, T. Veikkola, S. Mustjoki, T. Karpanen, B. Catimel, E. C. Nice, L. Wise, A. Mercer, H. Kowalski, D. Kerjaschki, S. A. Stacker, M. G. Achen and K. Alitalo, Isolated lymphatic endothelial cells transduce growth, survival and migratory signals via the VEGF-C/D receptor VEGFR-3, EMBO J. 20 (2001) 4762-4773; https://doi.org/10.1093/emboj/20.17.4762 